\title{
Experimental Study on Hydrocarbon Liquids Migration in Double-Porosity Medium Using Digital Image Analysis
}

\author{
Norhan Abd Rahman ${ }^{1 *}$, Loke Kok Foong ${ }^{2}$ \\ ${ }^{1}$ Centre of Tropical Geoengineering, Faculty of Civil Engineering, Universiti Teknologi Malaysia, Johor, Malaysia \\ ${ }^{2}$ Faculty of Civil Engineering, Universiti Teknologi Malaysia, Johor, Malaysia \\ *Corresponding author: norhan@utm.my
}

\begin{abstract}
The development activity of the country has played a part in natural disasters and climate change such as earthquake, El-Nino, tsunamis and water pollution have caused negative impact on human health and geo-environment. More complicated problems arise when the subsurface has experienced earthquake vibration, which no doubt influences the migration of hydrocarbon liquid into the groundwater sources. These problems need to be addressed in ensuring sustainable groundwater utilization. This paper aims to study the characteristics of hydrocarbon liquid migration that are important for the remediation cleanup of contaminated groundwater. The danger of reproductive toxic hydrocarbon chemicals has made actual on-site study infeasible and has been more practically replaced by physical model simulations. For this purpose, a physical laboratory experimental study was conducted to investigate the pattern and characteristics of different quantity toluene hydrocarbon migration in double-porosity medium under the vibration effect by using digital image analysis. The results of the experiments show that lower quantity of toluene hydrocarbon will take longer time to migrate to the bottom compared to higher quantity of toluene hydrocarbon. During experiment, air bubbles were continuously observed at the soil surface of toluene reducing due to the wettability of the liquids in the soil sample and the air trapped between the fractured aggregate and intra-inter aggregate pores. This study indicate that the digital image analysis is capable to provide the hydrocarbon flow rate and useful information for researchers and professionals to comprehensively understand migration characteristic.
\end{abstract}

Keywords_Kaolin Soil, Vibration, Contamination, Migration, Digital Image Processing Technique.

\section{INTRODUCTION}

$\mathrm{E}$ arthquake events recently reported at Tawau, Sabah [1]

and climate change had led to a negative impact on health issues and groundwater resources. These occurrences have called attention to the effect of vibration and contaminants on subsurface systems. In many countries, the critical issues of groundwater contamination in subsurface system is a problem that must be overcome. The leakage and spillage of hydrocarbon liquid can contribute to the contamination into the groundwater, rendering the quality of groundwater unsafe for drinking and industry usage. The groundwater aquifer would be more complicated under the effect of vibration on double-porosity medium. The ground failure and fractured soil deformation was caused by the vibration effect [2]. A greater understanding with respect to hydrocarbon liquid migration under the phenomena of fractured double-porosity soil required. The hydrocarbon liquid involves types of petroleum such as toluene, which has been used in this study.

The flow rate and phenomena of hydrocarbon liquid migration was affected by the soil structure. Normally, soils have different soil structure and non-homogeneous characteristics. Reduced intact soil shear strength and increased hydraulic conductivity are characteristics of cracked soil [3]. Krisnanto et al. [4] identified that cracked soil played a significant role in the liquid flow through deformable soil. It is widely acknowledged that the double-porosity media in usual condition is known as soil that displays two specific scales of porous media [5]. Double-porosity soil media is used to characterize soils that consist of two specific sub-regions with contrasting hydraulic properties that display different characteristics of pore sizes [6]. Previous research by Lakeland et al. [7] had stated that double-porosity could be formed through the earthquake vibration effect in which loosely packed water-saturated granular soils (sands and soil) are subjected to strong earthquake shaking, where they may liquefy and cause large deformations of soil with great destructive power. In earthquake engineering theory, the bedrock motion with highest response is the Peak Ground Acceleration (PGA) and the free surface motion with highest response is the Peak Surface Acceleration (PSA). The acceleration indicate either amplify or dis-amplify, based on the Eurocode 8 Part 1 [25] for classifying five ground types (A to E) and characterization of the site [2]. Therefore, this study used ground response analysis to identify the soil sample by the propagation of ground motion to the surface because in practice, ground response analysis was used to determine the crack and ground type.

Lewandowska et al. [8] discovered that soil in the laboratory can be used to create double-porosity for one-dimensional infiltration experiments. Furthermore, laboratory prepared double-porosity kaolin soil for one-dimensional drying and consolidation experiments was performed by Bagherieh et al. [9]. Recently, the physical experiments on double-porosity soil have begun to increase and researchers such as (Sa'ari et al. [10]; Loke et al. [11]; Tran-Ngoc et al. [12]; Kamaruddin et al. [13]; Alazaiza et al. [14]; and Ngien et al. [15]) have 
contributed an understanding of the soil characteristics in double-porosity soil. However, the studies were limited to intact double-porosity without applying any vibration effect on the double-porosity soil. To the best of our knowledge and upon reviewing other research papers, experiments on fractured double-porosity soil under vibration effect still have a gap to evaluate and investigate.

Digital image processing technique was used to understand and analyze the liquid migration in fractured double-porosity soil. Normal ways to monitor and observe the liquids migration require a lot of measuring apparatus, which may interfere with the sample original setup; however, digital image processing technique can overcome the problem using nondestructive and nonintrusive techniques. Based on the overview by Agaoglu et al. [16], the experiment involved noninvasive imaging techniques which used observation and characterization of multiphase system for greater precision. In this case, a number of researchers [13]-[15] and [17]-[23] have carried out the nonintrusive image techniques for experiment of liquid migration analysis. Therefore, the porosity and cracked soils are difficult to observe by naked eye and for this argument, digital image processing technique was acceptable for use in this study to investigate and observe hydrocarbon liquid migration in fractured double-porosity soil.

In addition, Ngien et al. [24] asserted that the problem of hydrocarbon contamination in the subsurface as well as the difficulty gathering reliable data concerning hydrocarbon liquid migration behaviour, in particular the laboratory experiments, will ensure the continuity of effort to understand, observe and overcome such problem. Therefore, a laboratory experimental model was conducted to investigate the behaviour of hydrocarbon liquid migration in fractured doubleporosity soil by using digital image processing technique. Thus, to accomplish the aims of this study, the objectives are given in the following (i) to investigate the vibration responses of the fracture double-porosity soil, (ii) to determine the characteristic of hydrocarbon liquid migration in fractured double-porosity soil using digital image processing technique, (iii) to differentiate the flow rate and phenomena of hydrocarbon liquid migration in fractured double-porosity. Essentially, this study covered the physical experiment model with double-porosity aggregated soil vibrated by using a vibratory table involving a special assemble acrylic glass soil column and accelerometer to obtain the vibration responses. The hydrocarbon liquid migrations were observed in a $100 \mathrm{~mm}$ height fractured soil sample in acrylic glass circular column. The aggregated soil sample mixed with $25 \%$ water was added to dry kaolin S300 soil. Toluene was used as a hydrocarbon liquid source. Red dye oil was used to dye the Toluene to increase the visualization of liquid migration. Dyed Toluene with $70 \mathrm{ml}$ and $150 \mathrm{ml}$ was poured instantaneously on top of the soil surface for each aggregated soil sample. The image analysis was accomplished by using Surfer programme version 10 and Matlab routine to analyse the liquid migration pattern, behaviour and migration flow rate.

\section{METHOD}

The laboratory soil sample preparation, laboratory experiment setup and procedure, and laboratory digital image processing setup used in this study were briefly discussed in subsequent sections.

\section{A. Laboratory Soil Sample Preparation}

The soil sample material used in this study to create doubleporosity was commercially available kaolin soil S300. The kaolin soil properties was tested based on British Standard BS 1377-2:1990 and BS 1377-5:1990 to obtain liquid limit = $41 \%$, plastic limit $=27.5 \%$, plasticity index $=13.5 \%$, particle density $=2.66 \mathrm{Mg} / \mathrm{m}^{3}$, particle size distribution $=(4 \%$ sand $93 \%$ silt; $4 \%$ clay) and falling head permeability test ( $\left.\mathrm{K}_{\text {average }}\right)$ $=5.41 \times 10^{-9}$. The kaolin soil S300 was classified under the Unified Soil Classification System (USCS) as silt with low plasticity (ML) based on the value of Atterberg limits and particle size distribution.

As previously explained, the method established by Bagheriah et al. [9] was used to prepare the aggregated soil sample. The dried kaolin powder was first mixed with $25 \%$ of moisture content to prepare the aggregated soil sample for sample 1 and sample 2 , respectively. The selection of moisture content in this experiment was based on the liquid limit, and the optimum moisture content for kaolin soil sample in this study was $26.5 \%$. Thereafter, in a cool condition with minimum 24 hours, the mixed sample was cured with kept in a re-sealable plastic bag for the purpose to maintain the moisture content from being evaporated. After that, the soil mixtures that passed $2.36 \mathrm{~mm}$ sieve for both samples 1 and 2 were placed in acrylic soil column and compressed to a height of $100 \mathrm{~mm}$ using a compression machine. The acrylic soil column with dimension of $300 \mathrm{~mm}$ high $\mathrm{x}$ $100 \mathrm{~mm}$-outer diameter and $94 \mathrm{~mm}$-inner diameter has been chosen to monitor and observe the changes occurring inside the whole area of circular column. The prepared aggregated soil sample is shown in Figure 1.

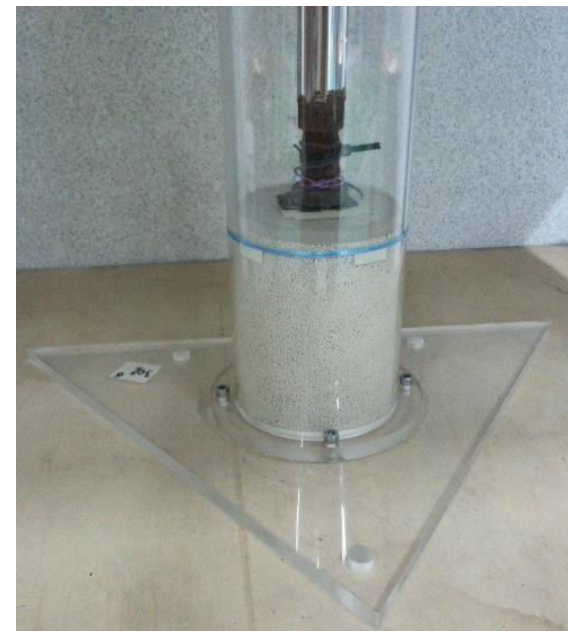

Figure 1. Prepared kaolin soil sample

The physical laboratory experiments were implemented in special design circular acrylic soil column with aggregated soil sample fitted and bolted on the vibratory table in order to prevent any freedom of movement of the acrylic soil column. The vibratory table was used to vibrate soil sample with different vibration frequencies for the deformation process. In addition, to ensure that the vibration table functioned well, the vibration table frequencies must be calibrated by using a high sensitivity 
accelerometer together with Dewesoft Sirius System data-logger to check and obtain excitation frequency. This study used the vibration table excitation frequency in the range of $0.29 \mathrm{~Hz}$ to $0.98 \mathrm{~Hz}$. Soft plasticines with strong bonding behavior were used as a coupling system between accelerometer and soil, while another accelerometer has been attached to the vibratory table with the same procedure. The view of vibratory table setup to vibrate the aggregated soil sample was developed by Loke et al. [2] as shown in Figure 2.

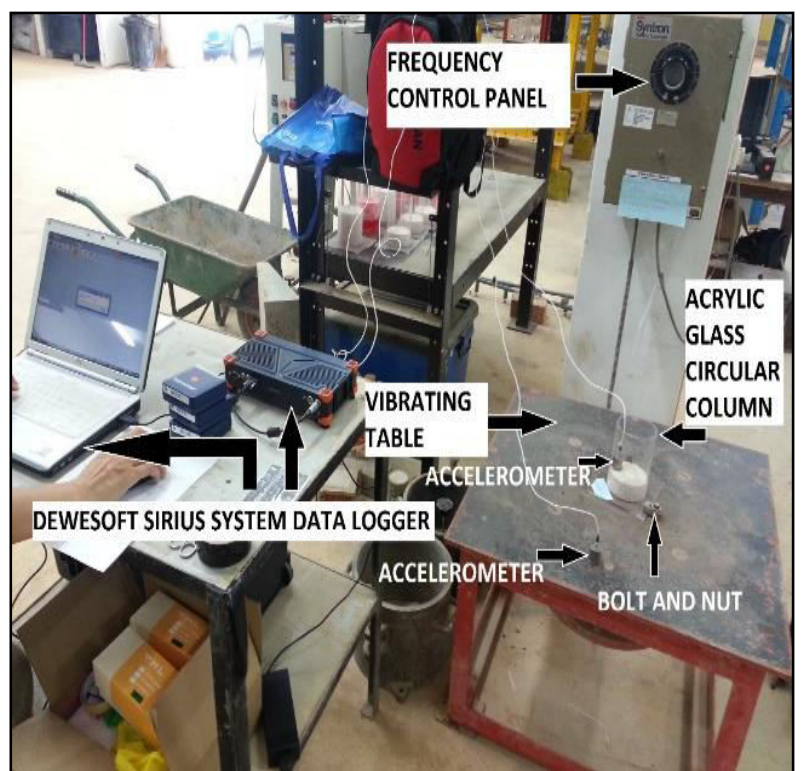

Figure 2. 3D diagram of vibratory table setup [2]

The results of fractured soil pattern with $25 \%$ moisture content for sample 1 and sample 2 before and after vibration process are displayed in Figure 3.

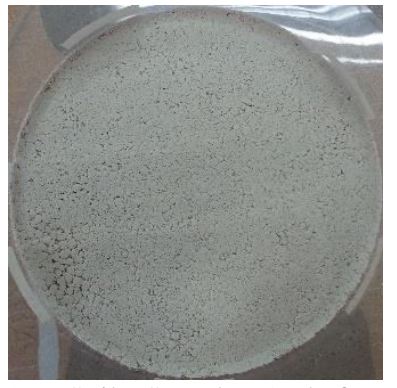

(a) Soil Sample 1 before vibration process

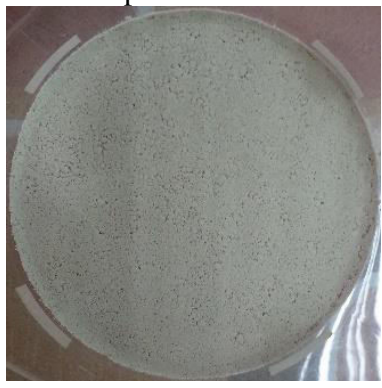

(c) Soil Sample 2 before vibration process

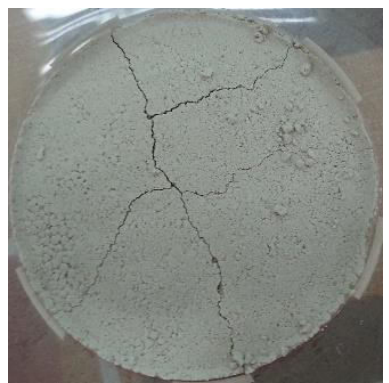

(b) Soil Sample 1 after vibration process

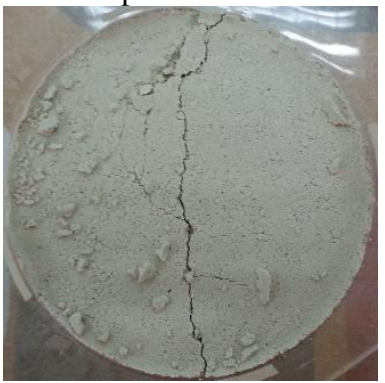

(d) Soil Sample 2 after vibration process

Figure 3 . The results of before and after vibration process for soil samples 1 and 2 with $25 \%$ moisture content

\section{B. Laboratory Experiment Setup and Procedure}

The acrylic soil column containing fractured double-porosity soil was used to monitor and measure the hydrocarbon liquid migration that occurs inside the whole circular column area with the aim of non-intrusion and non-destruction of the original soil sample setup. In each sample, the experimental measurement and setup of the $\mathrm{V}$ shape mirror and soil column position was arranged as shown in Figure 4 for hydrocarbon liquid migration image acquisition.

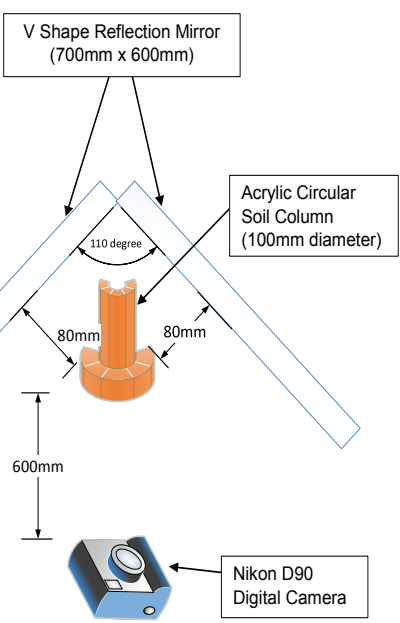

Figure 4. Digital image acquisition setup and measurement position

The main equipment for hydrocarbon liquid migration image acquisition system was the Nikon D90 DSLR digital camera and a V shape reflection mirror was used to reflect the whole image of circular column area. The Nikon D90 DSLR camera used medium size image format of $3216 \times 2136$ pixels and fixed with sensor size of $23.6 \mathrm{~mm} \times 15.8 \mathrm{~mm}$, resulting in each pixel having the size of $5.6 \times 5.6 \mu \mathrm{m}$. The ISO speed and aperture setup of digital camera during the experiments were set at 2500 and f/5.6, respectively, which has been implemented for all the soil sample experiments. The experiment used a V shape mirror common to allow $100 \%$ soil column surface exposure in a single view of image acquisition throughout the experiment. Thus, the images of the hydrocarbon liquid migration throughout the whole area of soil column circumference can be initially captured by just a single click on the digital camera. The light source for sample 1 and sample 2 come from linear fluorescent lamp-40 watt with output luminous flux of 2600 lumens/watt, which was placed slightly above the soil column.

Samples 1 and 2 were first sheathed in white paper with predrawn gridline $(20 \mathrm{mmx} 20 \mathrm{~mm})$ onto the soil column as a control point on the reference image. Both experiments began by instantaneously pouring the dyed toluene onto the top center of fractured aggregated soil sample in acrylic soil column. The quantity of $70 \mathrm{ml}$ and $150 \mathrm{ml}$ dyed toluene was used in sample 1 and sample 2, respectively. After the dyed toluene was poured and covered the whole surface area of the fractured soil sample, the first digital image of dyed toluene migration was taken in a room temperature of $23^{\circ} \mathrm{C}$. The subsequent digital images were taken at a specific time interval to capture the dyed toluene migration pattern. The subsequent digital images, totaled 109 images in 1800 seconds for sample 1 and 37 images in 110 seconds for sample 2. 


\section{Laboratory Digital Image Processing Setup}

The color digital images were captured and saved in the format of JPEG. The recorded images in JPEG format then were transferred from digital camera to computer for further image processing using Surfer Software and Matlab routine. The first step was digitization of the control points of area of interest on reference image to extract the image coordinates of control points. Area of interest refers to pre-determined migration boundary area $(\mathrm{V}$ shape refection image and front image) for sample 1 and sample 2, containing the dyed toluene as shown in Figure 5.

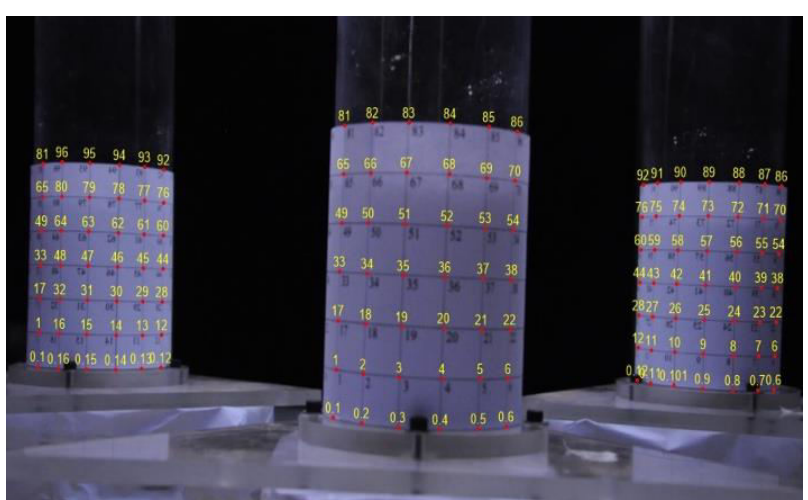

Figure 5. Control point position on reference image cover the area of interest for $\mathrm{V}$ shape reflection image and front image

After completing the image control point, Matlab routine was used to convert data from area of interest into red-green-blue (RGB) and hue saturation intensity (HSI) digital image format. The HSI value and RGB value from image were extracted and saved in text files using American Standard Code for Information Interchange format. The subsequent digital image was looped three times by using Matlab routine to extract and save the intensity values for all three section areas of interest ( $\mathrm{V}$ shape refection image count as sections one and two and front image counts as section three) of the circular acrylic soil column. Lastly, Surfer Software was used to plot the contour characteristic pattern of dyed toluene migration based on the HSI values. The HSI contour plot of hydrocarbon liquid migration behaviour can provide detailed information to facilitate researchers to understand the pattern of hydrocarbon liquid migration characteristic and the migration flow rate.

\section{RESULTS AND DISCUSSION}

The results of the soil surface acceleration and table acceleration for samples 1 and 2 with $25 \%$ moisture content are shown in Figure 6. Each frequency range has a different response, however only the peak acceleration has been chosen for discussion. The acceleration response result has shown the highest peak soil surface acceleration (PSA) value at $1.08 \mathrm{~g}$ and the lowest peak soil surface acceleration (PSA) value at $0.56 \mathrm{~g}$ for the overall frequency range with no obvious pattern to the vibratory table excitation frequency. The relationship could be seen to increase in relation to table acceleration. The observation shows the gradual increase of vibration table excitation frequency yielded different responses from the respective soils. Furthermore, during the observation for sample 1, the aggregated kaolin soil started to fracture during the frequency at $0.29 \mathrm{~Hz}$ and when the PSA was about $0.71 \mathrm{~g}$. The observation indicated that the accelerometer uprooted and was disconnected from the soil surface area as the aggregated kaolin soil was extremely fractured and the dilation process took place. The fractured soil sample displayed amplification shaking. Therefore, based on Eurocode 8 Part 1 [25] for classification of ground type, samples 1 and 2 have ground type $\mathrm{D}$, in which the soil has a soft-tofirm cohesiveness, because soil samples 1 and 2 with $25 \%$ moisture content experienced amplification shaking during the vibration process.

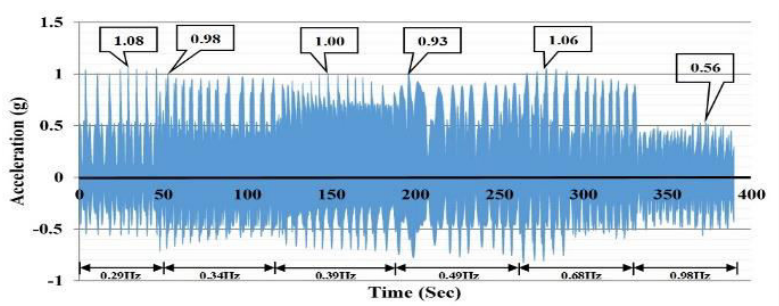

(a) Soil surface acceleration response (PSA)

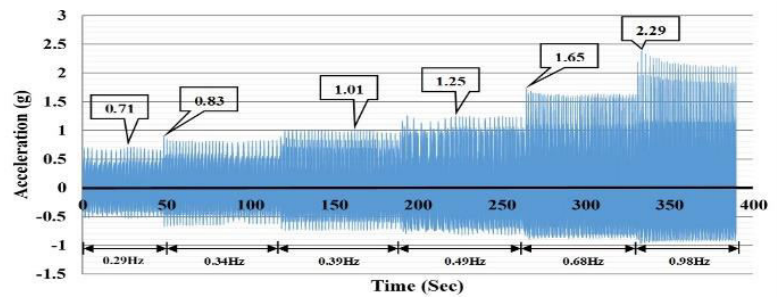

(b) Table acceleration response (PGA)

Figure 6. Results of acceleration response for soil samples 1 and 2

The results after the hydrocarbon liquid migration process for the top soil surface with the divided measurement of actual size column circumference zone for sample 1 and sample 2 to visualize the crack position is shown in Figure 7.

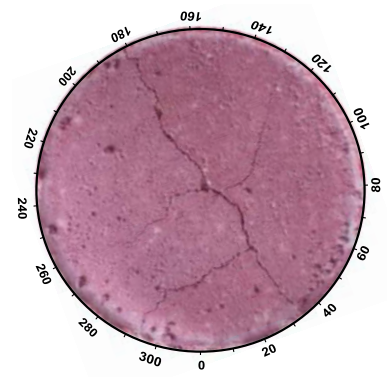

a) Soil sample $1(70 \mathrm{ml}$ Toluene)

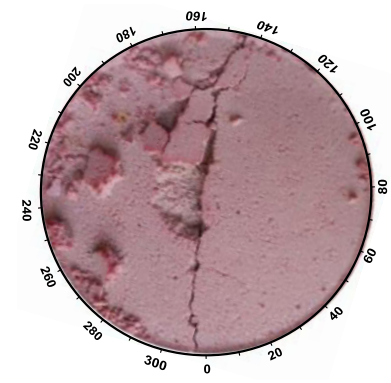

b) Soil sample $2(150 \mathrm{ml}$ Toluene)
Figure 7. Hydrocarbon liquid migrated soil surface with measurement of actual column circumference zone

The downward migration pattern of HSI plot of hydrocarbon liquid in the fractured double-porosity soil sample with $25 \%$ moisture content for sample 1 and sample 2, respectively, are shown in Figures 8 and 9. The HSI plots in two-dimensional shape are clearly apparent when the plot in curve join the left and right 
boundary which form a circular shape similar to soil column; however, the hydrocarbon liquid migration in the circular acrylic soil column was one-dimensional. Sample 1 used $70 \mathrm{ml}$ dyed toluene and sample 2 used $150 \mathrm{ml}$ dyed toluene poured instantaneously on top of the soil samples surface by using glass funnel to ensure that the dyed toluene downward migrated in one-dimension. In both samples, hydrocarbon liquid would have migrated into the kaolin soil sample before the whole soil sample surface was covered.
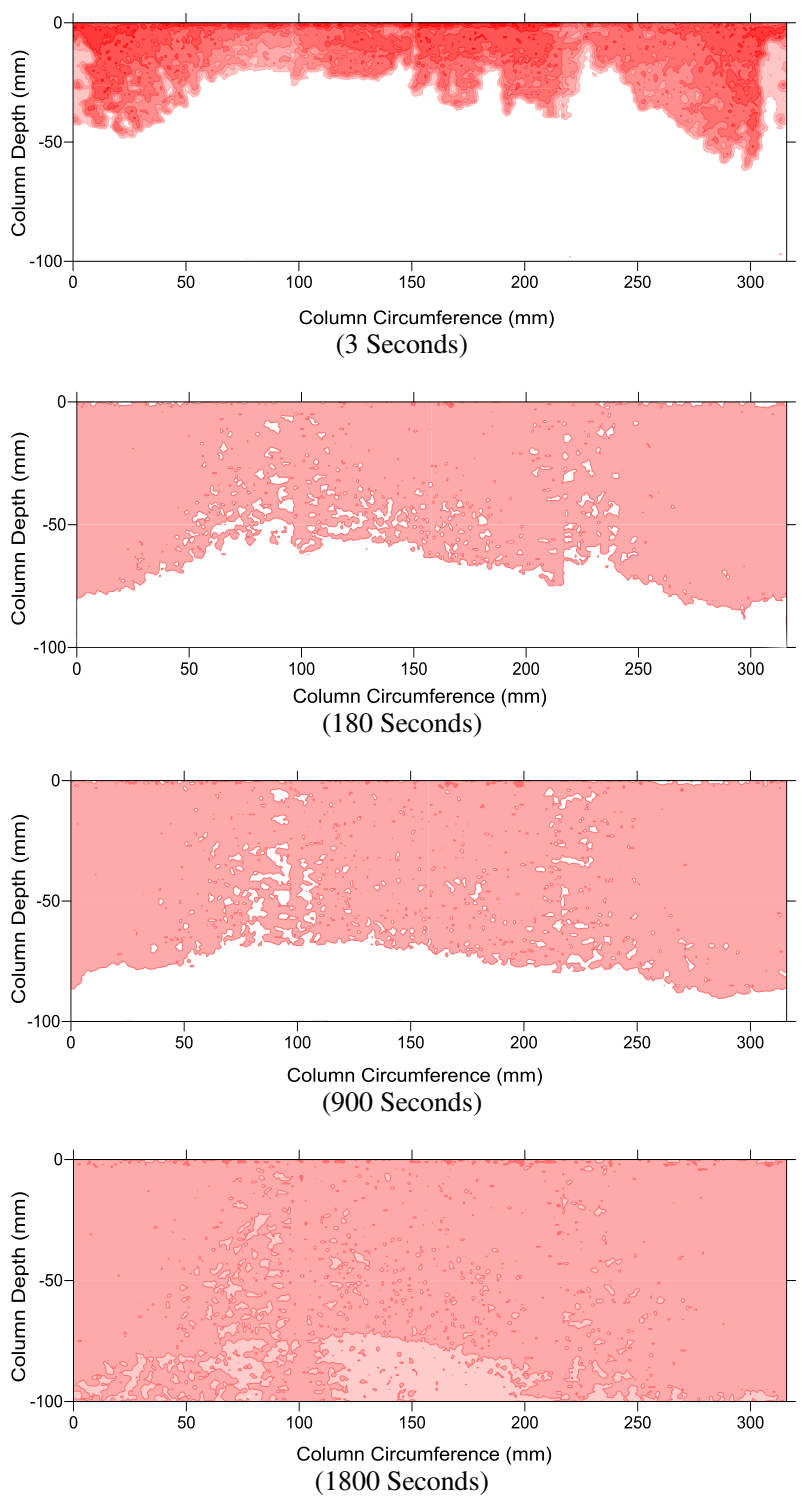

Figure 8. HSI plots of downward migration in fractured doubleporosity soil for $70 \mathrm{ml}$ dyed toluene

In sample 1, the selected HSI plots of hydrocarbon liquid migration recorded at intervals of 3, 180, 900, and 1800 seconds, respectively, were selected for dyed toluene migration HSI plots as can been seen in Figure 8. Based on observation and the HSI intensity contour plot results, the flow of dyed toluene migration was not consistently downward toward the front $\mathrm{x}$-axis horizontal line as the fractured doubleporosity soil was non-homogenous. Rapid migration occurred at the cracked soil surface condition compared to locations that were not cracked in soil surface as shown in Figure 7a. For the dyed toluene to completely recede from view on the whole top soil surface area into the fractured soil sample of the test took about 3 seconds, which is a very fast speed. Meanwhile, the overall duration for dyed toluene migration from the top surface to the bottom was 1800 seconds and further observation at 3600 seconds showed no changes in migration pattern where the dyed toluene migration along the $\mathrm{x}$-axis reached $100 \%$ downward depth of the soil column. At 3 seconds after the commencement of the experiment, the dyed toluene migration reached halfway of the test sample at the location of fractured soil surface as shown in Figure 8. The hydrocarbon liquid with $70 \mathrm{ml}$ migrated to the bottom in 1800 seconds in this study because the capillary force exerted by the small quantity of the toluene was insufficient to pressure the migration to penetrate fast. In addition, the factor of the $25 \%$ soil moisture content was considered quite dry, which caused the dyed toluene to be absorbed by the soil and to fill the empty porosity.

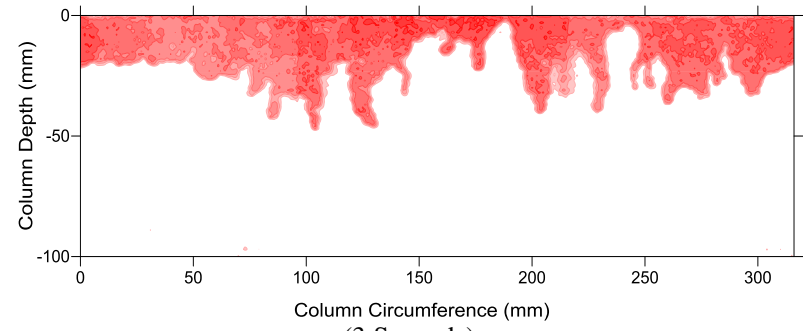

(3 Seconds)
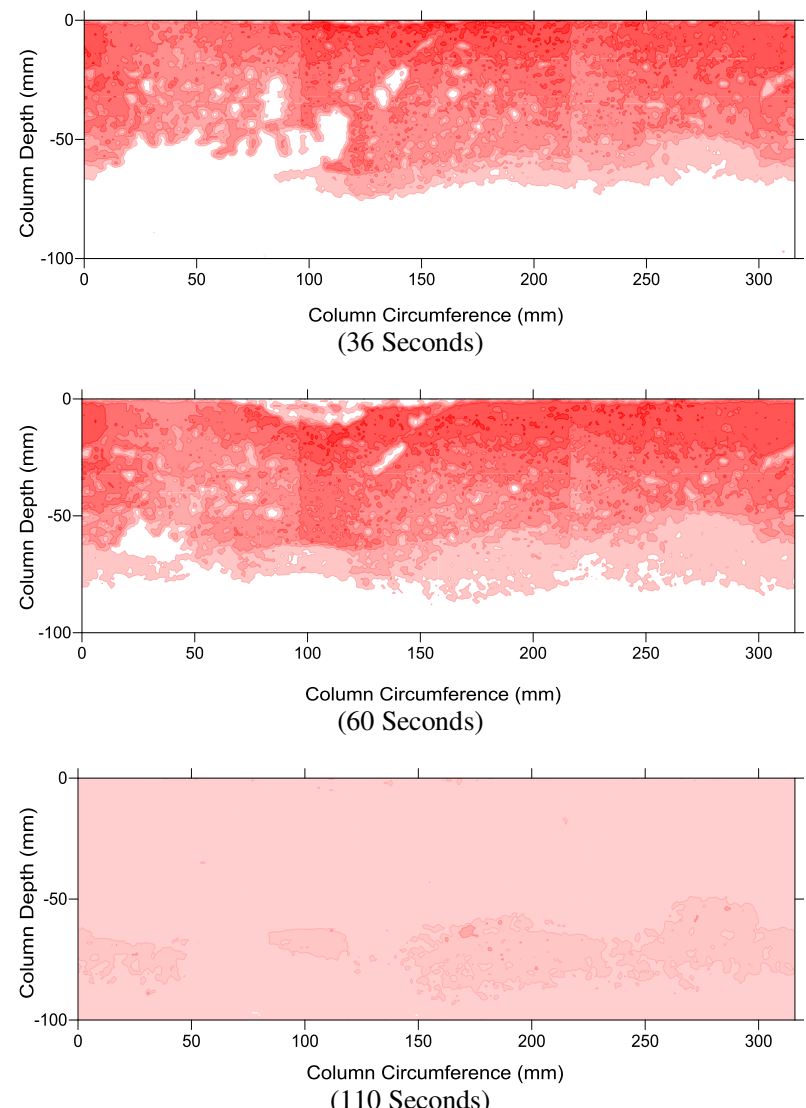

Figure 9. HSI plots of downward migration in fractured double-porosity soil for $150 \mathrm{ml}$ dyed toluene

In sample 2, the intervals of 3, 36, 60, 110 seconds, respectively, were selected for hydrocarbon liquid migration HSI plot as shown in Figure 9. The dyed toluene migration was similar to the result found in sample 1. The flow of dyed toluene migration was not uniformly downward at the front boundary horizontal 
line due to the non-homogeneity of the fractured doubleporosity soil structure. At 3 seconds after the initiation of the experiment, the dyed toluene migration reached halfway of the test sample at the location of fractured soil surface as shown in Figure 7b. The migration can be considered as one-dimensionally downward because the dyed toluene poured on top of the test sample had covered the whole sample surface. It took about 60 seconds for the dyed toluene to completely disappear from the whole top soil surface area into the fractured soil sample of the test. Meanwhile, the overall duration for dyed toluene migration from the top surface to the bottom was 110 seconds and further observation at 300 seconds showed no changes in migration pattern where the hydrocarbon liquid migration fully reached the bottom of the soil column. From the result, it can identified and differentiated that soil sample 2 had the faster migration compared to soil sample 1 as shorter time was taken for hydrocarbon liquid to reach the bottom of the soil column in soil sample 2 . Furthermore, the difference between samples 1 and 2 was in the migration behaviour and migration speed time. The sample 2 migration time was shorter because of the capillary force exerted by the dyed toluene pressure in top surface of the soil sample which had yet to migrate into the soil sample. Besides, the air bubbles were continuously observed at the soil surface of toluene reducing due to the wettability of the liquid in the soil sample.

The measured values of dyed toluene migration depth as a function of column circumference for selected critical time interval are shown in Figures 10 and 11 for sample 1 and 2, respectively. From Figure 10, the dyed toluene migrated fastest between 0 to $30 \mathrm{~mm}$ and 260 to $310 \mathrm{~mm}$ along the soil column circumference because of the cracked soil surface condition compared to other locations on the soil surface that were not cracked as shown in Figure 7a. The dyed toluene migration was dramatically expedited between 3 seconds and 180 seconds, which show extraordinary difference in migration pattern. This could be because the factor of dyed toluene pressure was high at the fractured doubleporosity soil, causing the hydrocarbon liquid to easily downward penetrate into the cracked soil sample.

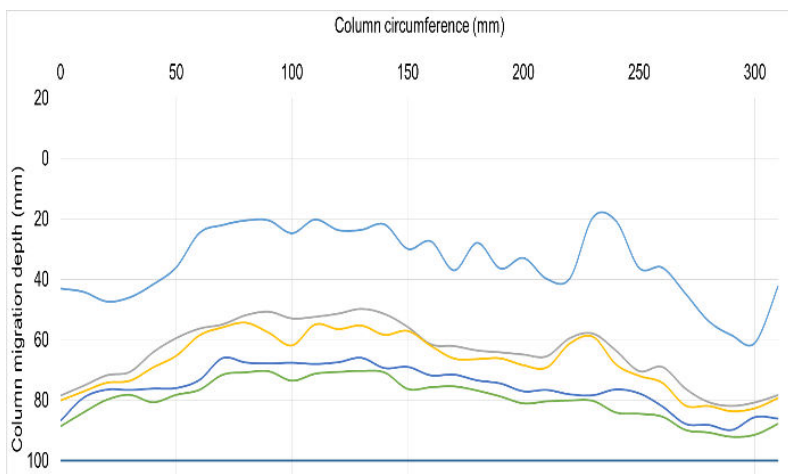

120

-3 Seconds -180 Seconds -300 Seconds -900 Seconds -1200 Seconds -1800 Seconds

Figure 10. Measured values of dyed toluene migration depth as a function of column circumference for selected critical time interval in sample 1
Based on Figure 11, the dyed toluene migration was fastest between 100 to $150 \mathrm{~mm}$ and 250 to $300 \mathrm{~mm}$ along the soil column circumference. The measured actual column circumference zone as shown in Figure $7 \mathrm{~b}$ indicated the soil surface crack position. The dyed toluene migration was expedited between 60 seconds and 90 seconds, which indicate significant changes in migration pattern. This phenomena was similar to sample 1 , where the influential factors were the capillary force exerted by the dyed toluene and high flow water pressure at the condition where the soil samples were fractured and cracked. Therefore, the mentioned factor caused the dyed toluene migration to penetrate downward into the soil sample easier and faster.

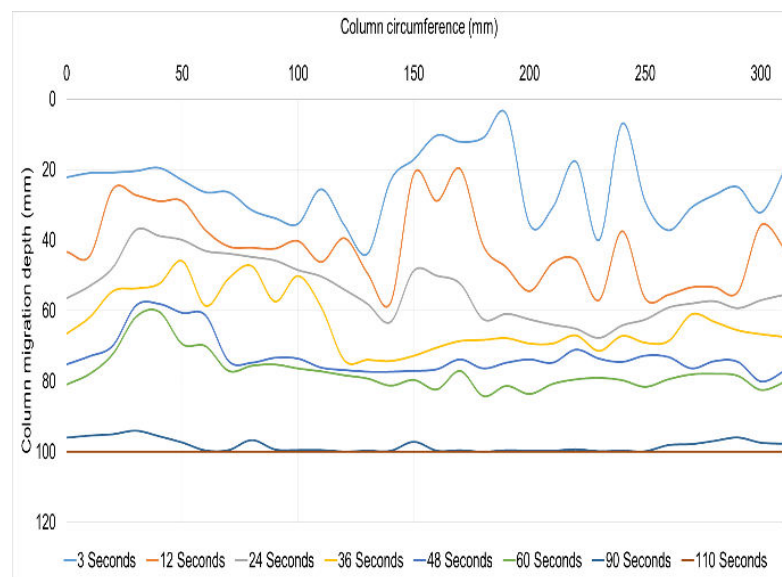

Figure 11. Measured values of dyed toluene migration depth as a function of column circumference for selected critical time interval in sample 2

The calculated migration flow rate for the higher and overall average speed for every $60 \mathrm{~mm}$ column circumference zone was presented in Table 1. Sample 1 shows the higher migration flow rate from initial to 3 seconds was at $300 \mathrm{~mm}$ column circumference zone with the migration flow rate of $61.025 \mathrm{~mm} / \mathrm{s}$. Meanwhile, the other high migration flow rates occurred at $0 \mathrm{~mm}$ and $180 \mathrm{~mm}$ column circumference with the value of 43.063 $\mathrm{mm} / \mathrm{s}$ and $27.935 \mathrm{~mm} / \mathrm{s}$, respectively. Thus, the overall average dyed toluene migration flow rate for sample 1 is $5.607 \mathrm{~mm} / \mathrm{s}$. The highest average migration flow rate was at the $300 \mathrm{~mm}$ column circumference zone at 10.182 $\mathrm{mm} / \mathrm{s}$. This could be because the larger fractured and cracked soil structure after the vibration was at the position of $300 \mathrm{~mm}$.

Sample 2 displays the higher migration flow rate from initial to 3 seconds at $120 \mathrm{~mm}$ column circumference zone with the migration flow rate of $35.785 \mathrm{~mm} / \mathrm{s}$. Meanwhile, the column circumference zone at $300 \mathrm{~mm}$ and $60 \mathrm{~mm}$ also have a high migration speed rate with the values of $32.146 \mathrm{~mm} / \mathrm{s}$ and $26.404 \mathrm{~mm} / \mathrm{s}$, respectively. The higher migration speed rate was due to the cracked or fractured at soil sample surface as shown in Figure $7 \mathrm{~b}$ for the actual column circumference. Therefore, the overall average dyed toluene migration flow rate for sample 2 is $6.813 \mathrm{~mm} / \mathrm{s}$. This scenario occurred because the higher quantity of the toluene in sample 2 has caused the greatest pressure on top of the soil surface, forcing penetration to be faster than sample 1 . 
Table 1. Migration flow rate for every $60 \mathrm{~mm}$ column circumference

\begin{tabular}{lcc|cc}
\hline Column & \multicolumn{3}{c}{ Migration Flow Rate $(\mathrm{mm} / \mathrm{s})$} \\
\cline { 2 - 5 } $\begin{array}{l}\text { Circumference } \\
\text { Zone }(\mathrm{mm})\end{array}$ & \multicolumn{2}{c}{ Soil sample 1 (70ml) } & \multicolumn{2}{c}{$\begin{array}{c}\text { Soil sample 2 } \\
(150 \mathrm{ml})\end{array}$} \\
\cline { 2 - 5 } & Higher & Average & Higher & Average \\
& Flow & Flow for & Flow & Flow for \\
& Between & All the & Between & All the \\
& Initial to & Time & Initial to & Time \\
& 3 & Interval & 3 & Interval \\
& Seconds & & Seconds & \\
\hline 0 & 43.063 & 7.188 & 22.169 & 6.937 \\
60 & 24.685 & 4.135 & 26.404 & 7.873 \\
120 & 23.733 & 3.982 & 35.785 & 9.895 \\
180 & 27.935 & 4.676 & 10.948 & 3.834 \\
240 & 20.732 & 3.478 & 7.568 & 3.704 \\
300 & 61.025 & 10.182 & 32.146 & 8.637 \\
\hline
\end{tabular}

From the result, it can be concluded that the sample 1 column circumference positions at $300 \mathrm{~mm}, 0 \mathrm{~mm}$, and $180 \mathrm{~mm}$ demonstrated faster migration flow and the faster migration flow for sample 2 was at column circumference positions $120 \mathrm{~mm}, 300 \mathrm{~mm}$, and $60 \mathrm{~mm}$ as shown in Figure 7. The average migration flow rate for soil sample 2 is faster than soil sample 1. From the experiment result, the factors that significantly influenced the hydrocarbon liquid migration in soil samples 1 and 2 was the fractured pattern of the soil sample, porosity of the soil sample, the quantity of the liquid and the capillary pressure of the liquid.

\section{CONCLUSION}

The laboratory experiment on hydrocarbon liquid migration in fractured double-porosity soil with $25 \%$ moisture content had been carried out. This purpose of the experiment was to investigate the vibration of soil sample and hydrocarbon liquid behaviour as well as to differentiate the characteristic and flow rate in the fractured double-porosity soil in circular soil column. The digital image processing technique using Surfer Software and Matlab routine was applied to extract and analyze the hydrocarbon liquid migration data obtained from captured digital image. The laboratory experiment successfully provided the results of acceleration response and various behaviours of migration and also differentiated the characteristic and flow rate of hydrocarbon migration at $25 \%$ moisture content. From the results observed, both samples have been classified as ground type $\mathrm{D}$, in which the soil has a soft-to-firm cohesiveness structure. The sample 2 hydrocarbon liquid migrated faster from top to the bottom of soil column compared to sample 1. Meanwhile, samples 1 and 2 have $100 \%$ fully migrated to the bottom of the soil column. Furthermore, the sample 2 flow rate with $6.813 \mathrm{~mm} / \mathrm{s}$ was higher compared to APL flow rate with $5.607 \mathrm{~mm} / \mathrm{s}$. The results proved that the factors that significantly influenced the hydrocarbon liquid migration in soil sample 1 and soil sample 2 was the fractured pattern of the soil sample, the quantity of the liquid, the structure of the soil sample, the porosity of the soil sample, the wettability of liquid in the soil sample and the capillary pressure of liquid. In conclusion, the acceleration response of soil sample and hydrocarbon liquid migration contour plots and HSI intensity value have provided detailed useful information to researchers and professionals to comprehensively understand and simulate the behaviour and pattern of toluene migration that could be used to identify the remediation method and to ensure the sustainability of groundwater resources.

\section{ACKNOWLEDGMENTS}

This study was supported by the Research Management Centre (RMC), Universiti Teknologi Malaysia under Research University Grant - Tier 1 (PY/2016/06547) from the Ministry of Higher Education Malaysia. The authors would also like to thank their respective University, Public Service Department Malaysia, Geotechnical Laboratory, Hydraulic and Hydrology Laboratory, Engineering Seismology and Earthquake Engineering Research Group (eSEER), and Survey Unit, Faculty of Civil Engineering, Universiti Teknologi Malaysia for kind assistance lent to this research. The second author was supported through the federal training award by the Public Service Department under Prime Minister's Department, Malaysia.

\section{REFERENCES}

[1] L. Stephanie. (2015, Dec 22). Tawau Residents Shaken by Quake (The Star) [Online]. Available http://www.thestar.com.my/news/nation/2015/12/22/tawauresidents -shaken-by-quake-early-morning-tarakan-temblorsends-people-running-out-of-homes/.

[2] K. F. Loke, N. A. Rahman and R. Nazir, "Experimental study on unsaturated double-porosity soil phenomena under vibration effect," J. Teknologi, vol. 79(4), pp. 65-72, 2017.

[3] D. G. Fredlund, S. L. Houston, Q. Nguyen and M. D. Fredlund, "Moisture movement through cracked clay soil profiles," Geotechnical and Geological Engineering, vol. 28(6), pp. 865-888, 2010.

[4] S. Krisnanto, H. Rahardjo, D. G. Fredlund and E. C. Leong, "Mapping of cracked soils and lateral water flow characteristics through a network of cracks,". Eng. Geology, vol. 172, pp. 12-25, 2014.

[5] A. Carminati, A. Kaestner, P. Lehman and H. Fluhler, "Unsaturated water flow across soil aggregate contacts,". Adv. In Water Resources, vol. 31(9), pp. 1221-1232, 2008.

[6] S. K. Ngien, N. A. Rahman, M. M. Bob, K. Ahmad, R. Sa'ari and R. W. Lewis, "Observation of light non-aqueous phase liquid migration in aggregated soil using image analysis," Transport in Porous Media, vol. 92(1), pp. 83100, 2011.

[7] D. L. Lakeland, A. Rechenmacher and R. Ghanem, "Towards a complete model of soil liquefaction: the importance of fluid flow and grain motion," Proc. of the Roy. Soc. A: Math., Physical and Eng. Sci., London, 2014, A470:20130453.

[8] J. Lewandowska, A. Szymkiewicz, W. Gorczewska and M. Vauclin, "Infiltration in a double-porosity medium: Experiments and comparison with a theoretical model," Water Resources Research, vol. 41(2), pp. W02022, 2005.

[9] A. R. Bagherieh, N. Khalili, G. Habibagahi and A. Ghahramani, "Drying response and effective stress in a double porosity aggregated soil," Eng. Geology, vol. 105(1-2), pp. 44-50, 2009.

[10] R. Sa ari, N. A. Rahman, N. H. Latif Abdul, Z. M. Yusof, S. K. Ngien, S. A. Kamaruddin, M. Mustaffar and 
M. A. Hezmi, "Application of digital image processing technique in monitoring LNAPL migration in double porosity soil column," J. Teknologi, vol. 3(72), pp. 23-29, 2015.

[11] K. F. Loke, N. A. Rahman and M. Z. Ramli, "A laboratory study of vibration effect for deformable double-porosity soil with different moisture content," Malaysian J. of Civil Eng., vol. 28 SI(3), pp. 207-222, 2016.

[10] G. I. Barenblatt, P. I. Zheltov and I. N. Kochina, "Basic concepts in the theory of seepage of homogeneous liquids in fissured rocks [strata]," J. of Applied Math. and Mechanics, vol. 24(5), pp. 1286-1303, 1960.

[12]T. D. Tran-Ngoc, J. Lewandowska and H. Bertin, "Experimental evidence of the double-porosity effects in geo-meterials," Acta Geophysica, vol. 62, pp. 642-655, 2014.

[13] S. A. Kamaruddin, W. N. A. Sulaiman, N. A. Rahman, M. P. Zakaria, M. Mustaffar and R. Sa'ari, "A review of laboratory and numerical simulations of hydrocarbons migration in subsurface environments," $J$. of Environmental Sci. and Technology, vol. 4(3), pp. 191214, 2011.

[14] M. Y. D. Alazaiza, S. K. Ngien, M. M. Bob, S. A. Kamaruddin and W. M. F. Ishak, "Influence of macropores on DNAPL migration in double-porosity soil using light transmission visualization method," Transport in Porous Media, vol. 117, pp. 103-123, 2017.

[15] S. K. Ngien, P. Q. Chin, M. Hasan, M. I. Ali, M. Y. M. Tadza and N. A. Rahman, "Image analysis of non-aqueous phase liquid migration in aggregated kaolin," ARPN J. of Eng. and Applied Sci., vol. 11(10), pp. 6393-6398, 2016.

[16] B. Agaoglu, N. K. Copty, T. Scheytt and R. Hinkelmann, "Interphase mass transfer between fluids in subsurface formations: A review," Advances in. Water Resources, vol. 79, pp. 162-194, 2015.

[17] Y. Teng, S. A. Stanier and S. M. Gourvenec, "Synchronised multi-scale image analysis of soil deformations," Int. J. of Physical Modelling in Geotechnics, vol. 17(1), pp. 53-71, 2017.
[18]C. A. Murray, N. A. Hoult and W. A. Take, "Dynamic measurement using digital image correlation," Int. J. of Physical Modelling in Geotechnics, vol. 17(1), pp. 41-52, 2017.

[19]Z. Peng, C. Duwig, P. Delmas, J. P. Gaudet, A. G. Strozzi, P. Charrier and H. Denis, "Visualization and characterization of heterogeneous water flow in doubleporosity media by means of X-ray computed tomography," Transport in Porous Media, vol. 110, pp. 543-564, 2015.

[20]G. Flores, T. Katsumi, T. Inui and M. Kamon, "A simplified image analysis method to study LNAPLs migration in porous media," Soil and Foundation, vol. 51, pp. 835-847, 2011.

[21]C. Kechavarzi, K. Soga, T. H. Illangasekare and P. Nikolopoulos, "Laboratory study of immiscible contaminant flow in unsaturated layered sands," Vadose Zone Journal, pp. 1-9, 2008.

[22] J. D. McNeil, G. A. Oldenborger and R. A. Chincariol, "Quantitative imaging of contaminant distributions in heterogeneous porous media laboratory experiments," J. of Contaminant Hydrology, vol. 84, pp. 36-54, 2006.

[23] C. J. G. Darnault, J. A. Throop, D. A. Dicarlo, A. Rimmer, T. S. Steenhuis and J. Y. Parianges, "Measurement of fluid contents by light transmission in three-phase-oil-water-air systems in sand," Water Resource Research, vol. 37, pp. 1859-1868, 1998.

[24] S. K. Ngien, N. A. Rahman, K. Ahmad and R. W. Lewis, "A review of experimental studies on double-porosity soils," Scientific Research and Essays, vol. 7(38), pp. 3243-3250, 2012.

[25] Eurocode 8, "Design of sturctures for earthquake resistance, Part 1: General rules, seismic actions and rules for building," European Committee for Standardzation, Brussels, Belgium. 2004. 